\title{
Bayesian Estimation under Constant-Stress Partially Accelerated Life Test for Pareto Distribution with Type-I Censoring
}

\author{
Ali A. Ismail ${ }^{\mathrm{a}, \mathrm{b}}$ \\ ${ }^{a}$ King Saud University, College of Science, Department of Statistics and Operations Research, P. O. \\ BOX 2455, Riyadh 11451, Saudi Arabia \\ ${ }^{\mathrm{b}}$ Cairo University, Faculty of Economics \& Political Science, Department of Statistics, Giza 12613, \\ Egypt \\ aismail100@yahoo.com (Ali A. Ismail)
}

УДК 539.4

\section{Применение метода Байеса при планировании частично ускоренных ресурсных испытаний при постоянных нагрузках и цензурировании по времени типа I для распределения Парето}

\author{
Али А. Исмаил ${ }^{\mathrm{a}, \boldsymbol{\sigma}}$ \\ а Университет им. короля Сауда, Эр-Риад, Саудовская Аравия \\ ${ }^{\sigma}$ Каирский университет, Гиза, Египет
}

Исследуются вероятность параметров модели и применение метода Байеса для модели частично ускоренных ресурсных испытаний при постоянных нагрузках и иензурировании по времени типа I для распределения Парето второго рода. Выполнен расчет максимальной вероятности и индикаторов Байеса для параметров модели. С использованием метода аппроксимации Линдли получены апостериорные средние значения и вариации для квадратичной функиии потерь (ошибок). Показаны преимущества данной аппроксимации. Выполнено численное моделирование по методу Монте-Карло для образиов различных размеров и параметров модели для сравнительной оченки предложенных методов прогнозирования ресурса.

Ключевые слова: надежность, испытание, оценка максимальной вероятности, оценка по Байесу, квадратичная функция потерь.

Introduction. Accelerated testing ensures that specimens are exposed to elevated environmental conditions for fixed periods of time. Overstress testing consists of running a product at higher than normal levels of some accelerating stress(es) to shorting product life or to degrade product performance faster. Overstress constant stress testing is the most common form of Accelerated testing of specimens. Each specimen is tested under a constant stress level. Such testing scheme is easy and has numerous advantages Nelson [1].

As indicated by Ismail [2], accelerated life testing and partially accelerated life testing (PALT) are frequently used in modern reliability engineering to save time and cost. The aim of a PALT is to obtain more failure data in a restricted time without essentially using severe conditions to all test items.

Constant-stress PALT with type-I censoring were studied by some authors such as [3-6]. These studies had been made based on classical methods. This paper considers Lindley technique for estimating the parameters in constant-stress PALT. According to [7], such an approximation has numerous valuable applications especially for industrial fields. Also, in this respect, Achcar [8] said that "the use of approximate Bayesian methods could 
be a good alternative for the usual existing classical asymptotic methods used in accelerated life testing (ALT)."

There were some works on PALT in the context of Bayesian approach. For example, see Goel [9], DeGroot and Goel [10], Abdel-Ghani [11], Ismail [12]. The objective of this article is to use Lindley method to make a Bayesian analysis with a squared error loss function under time-censoring CSPALT. The Bayes estimators (BEs) of the acceleration factor and the distribution parameters are derived and compared with the maximum likelihood estimators (MLEs) counterparts by Monte Carlo simulations.

The rest of this paper is arranged as follows. In Section 1, the model and test method are described. Approximate BEs of the parameters under consideration are derived in Section 2. In Section 3 BEs derived in Section 2 are obtained numerically using Lindley's approximation and compared with the MLEs. Finally, Section 4 concludes the paper.

1. The Model and Test Method. The probability density function (PDF) of the Pareto distribution of the second kind is given by

$$
f_{T}(t ; \theta, \alpha)=\frac{\alpha \theta^{\alpha}}{(\theta+t)^{\alpha+1}}, \quad t>0, \theta>0, \alpha>0 .
$$

The survival function takes the form

$$
R(t)=\frac{\theta^{\alpha}}{(\theta+t)^{\alpha}},
$$

and the corresponding failure rate function is

$$
h(t)=\frac{\alpha}{\theta+t} .
$$

In a constant-stress PALT, $n \pi$ units randomly selected among $n$ test units sampled are allocated to severe condition and the remaining are allocated to normal condition. Each test item is tested until the censoring time is reached or the item fails.

The following assumptions are considered. r.v.'s.

1. The lifetimes $T_{i}, i=1, \ldots, n(1-\pi)$ of items allocated to use condition, are i.i.d.

2. The lifetimes $X_{j}, j=1, \ldots, n \pi$ of items allocated to accelerated condition, are i.i.d r.v.'s.

3. Suppose that the lifetime of an item at accelerated condition is denoted by $X$, then the lifetime of this item at use condition $T$ is given by the relation $T=\beta X$.

Since the lifetimes of the test items follow Pareto distribution of the second kind, the probability density function of an item tested at normal condition is given by (1).

The PDF under severe condition is expressed by

$$
f_{X}(x ; \theta, \alpha)=\frac{\beta \alpha \theta^{\alpha}}{(\theta+\beta x)^{\alpha+1}}, \quad x>0, \theta>0, \alpha>0,
$$

where $X=\beta^{-1} T$.

2. Bayesian Estimation. Here, Bayesian estimates are considered using non-informative priors via the technique of Lindley and the squared error (SE) loss function. The non-informative prior (NIP) for each parameter be represented by the limiting form of the appropriate natural conjugate prior. 
It follows that a NIP for the acceleration factor $\beta$ is given by

$$
\pi_{1}(\beta) \propto \beta^{-1}, \quad \beta>1
$$

Also, the NIP's for the scale parameter $\theta$ and the shape parameter $\alpha$ are, respectively, as

$$
\pi_{2}(\theta) \propto \theta^{-1}, \quad \theta>0 \quad \text { and } \quad \pi_{3}(\alpha) \propto \alpha^{-1}, \quad \alpha>0 .
$$

Therefore, the joint NIP of the three parameters can be expressed by

$$
\pi(\beta, \theta, \alpha) \propto(\beta \theta \alpha)^{-1}, \quad \beta>1, \theta>0, \alpha>0 .
$$

Via time-censored data, any unit can be tested at one condition only until a pre-fixed censoring time $\eta$ is attained. Therefore, the observed lifetimes $t_{(1)} \leq \ldots \leq t_{\left(n_{u}\right)} \leq \eta$ and $x_{(1)} \leq \ldots \leq x_{\left(n_{a}\right)} \leq \eta$ are ordered failure times at normal use and accelerated conditions, respectively, where $n_{u}$ and $n_{a}$ are the corresponding numbers of items failed in each stage. Let $\delta_{u i}$ and $\delta_{a j}$, be indicator functions such that $\delta_{u i} \equiv I\left(T_{i} \leq \eta\right)$ and $\delta_{a j} \equiv$ $\equiv I\left(X_{j} \leq \eta\right)$, where $i=1, \ldots, n$. Then, the overall likelihood function can be expressed by

$$
\begin{gathered}
L(\beta, \theta, \alpha)=\prod_{i=1}^{n(1-\pi)} L_{u i}\left(t_{i} ; \theta, \alpha\right) \prod_{j=1}^{n \pi} L_{a j}\left(x_{j} ; \beta, \theta, \alpha\right)= \\
=\prod_{i=1}^{n(1-\pi)}\left[\frac{\alpha \theta^{\alpha}}{\left(\theta+t_{i}\right)^{\alpha+1}}\right]^{\delta_{u i}}\left[\frac{\theta^{\alpha}}{(\theta+\eta)^{\alpha}}\right]^{\bar{\delta}_{u i}} \prod_{j=1}^{n \pi}\left[\frac{\beta \alpha \theta^{\alpha}}{\left(\theta+\beta x_{j}\right)^{\alpha+1}}\right]^{\delta_{a j}}\left[\frac{\theta^{\alpha}}{(\theta+\beta \eta)^{\alpha}}\right]^{\bar{\delta}_{a j}},
\end{gathered}
$$

where $L_{u i}$ is the likelihood function for $t_{i}$ at use condition, $L_{a j}$ is the likelihood function for $x_{j}$ at accelerated condition, $\pi$ is the proportion of sample units allocated to accelerated condition, and

$$
\bar{\delta}_{u i}=1-\delta_{u i} \quad \text { and } \quad \bar{\delta}_{a j}=1-\delta_{a j} .
$$

Using (6) and (7), the joint posterior distribution can be given by

$$
\begin{gathered}
g(\beta, \theta, \alpha \mid \underline{y}) \propto L(\underline{y} \mid \beta, \theta, \alpha) \pi(\beta, \theta, \alpha) \propto \\
\propto \frac{\beta^{n_{a}-1} \theta^{2 n \alpha-1} \alpha^{n_{u}+n_{a}}}{(\theta+\eta)^{\left(n \bar{\pi}-n_{u}\right) \alpha}(\theta+\beta \eta)^{\left(n \pi-n_{a}\right) \alpha}}\left[\prod_{i=1}^{n \bar{\pi}} \frac{1}{\left(\theta+t_{i}\right)^{\alpha+1}}\right]^{\delta_{u i}}\left[\prod_{j=1}^{n \pi} \frac{1}{\left(\theta+\beta x_{j}\right)^{\alpha+1}}\right]^{\delta_{a j}} .
\end{gathered}
$$

To obtain the posterior means and posterior variances of $\beta, \theta$, and $\alpha$, an approximation due to Lindley [13] is used.

Now, let $\Theta$ be a set of parameters $\left\{\Theta_{1}, \Theta_{2}, \ldots, \Theta_{m}\right\}$, where $m$ is the number of parameters, then the posterior expectation of an arbitrary function $u(\Theta)$ can be asymptotically estimated by 


$$
\begin{gathered}
E(u(\Theta))=\frac{\int_{\Theta} u(\Theta) \pi(\Theta) e^{\ln L(y \mid \Theta)} d \Theta}{\int_{\Theta} \pi(\Theta) e^{\ln L(y \mid(\Theta)} d \Theta} \approx \\
\approx\left[u+(1 / 2) \sum_{i, j}\left(u_{i j}^{(2)}+2 u_{i}^{(1)} \rho_{j}^{(1)}\right) \sigma_{i j}+(1 / 2) \sum_{i, j, k, s} L_{i j k}^{(3)} \sigma_{i j} \sigma_{k s} u_{s}^{(1)}\right] \downarrow \hat{\Theta},
\end{gathered}
$$

which is the Bayes estimator of $u(\Theta)$ under a squared error loss function, where $\pi(\Theta)$ is the prior distribution of $\Theta, u \equiv u(\Theta), L \equiv L(\Theta)$ is the likelihood function, $\rho \equiv \rho(\Theta)=$ $=\log \pi(\Theta), \sigma_{i j}$ are the elements of the inverse of the asymptotic Fisher's information matrix of $\beta, \theta$, and $\alpha$, and

$$
u_{i}^{(1)}=\frac{\partial u}{\partial \Theta_{i}}, \quad u_{i j}^{(2)}=\frac{\partial^{2} u}{\partial \Theta_{i} \partial \Theta_{j}}, \quad \rho_{j}^{(1)}=\frac{\partial \log \pi(\Theta)}{\partial \Theta_{j}}, \quad \text { and } \quad L_{i j k}^{(3)}=\frac{\partial^{3} \ln L(y \mid \Theta)}{\partial \Theta_{i} \partial \Theta_{j} \partial \Theta_{k}} .
$$

According to Green [14], the above posterior expectation is "very good and operational approximation for the ratio of multi-dimension integrals." Also, according to [7], it has important applied aspect. Some mathematical details are given at the end of this paper.

3. Monte Carlo Simulation studies. In this section, we illustrate the use of Bayesian approach via Lindley method for approximation of integrals to derive the marginal posterior moments of interest in the case of constant-stress PALT under type-I censoring. The data are generated from Pareto distribution with different sample sizes. For each sample size, 5,000 samples are obtained randomly. The posterior means and posterior variances of the three parameters are obtained numerically. In addition, the ML estimators and Bayes estimators are compared with respect to the mean squared errors (MSEs) and variability.

To assess and compare the performance of the MLEs and proposed estimators with the Lindley method, we perform simulation comparisons with data generated via various scenarios. Four numerical examples are provided with equal and unequal proportions of allocation for illustration. One of the considered populations is set the combination of $(\beta, \theta, \alpha)$ at $(3,0.8,0.5)$ with equal proportion of allocation $\pi=0.50$ with results reported in Table 1. A second combination is set at $(2,1.2,1.5)$ using also equal proportion of allocation $(\pi=0.50)$ with results shown in Table 2 . While the third combination is taken as $(3,0.8,0.5)$ based on unequal proportion of allocation $(\pi=0.30)$ with numerical results reported in Table 3. Concerning the fourth scenario, the combination is $(2,1.2,1.5)$ based on proportion of allocation set at $\pi=0.70$ with numerical results displayed in Table 4 .

Also, concerning the comparison between Bayesian estimators and the likelihood ones, the results have the same trend when unequal proportions of allocation are used. But, with larger proportion of allocation to the accelerated condition, it is noticed that Lindley method is much better than the likelihood-based method.

4. Some Main Remarks and Further Studies. In this paper both ML and Bayes estimations of the CSPALT model parameters have been presented using time-censored samples from Pareto distribution. The Bayes estimators have been considered under the assumptions of squared error loss functions and non-informative priors. Lindley's technique has been used to obtain the Bayesian estimates numerically. It has been found that the technique works very well even for small sample sizes. Also, it has been noted that Lindley's technique frequently produces posterior variances smaller than the variances of the maximum likelihood estimators. So, it gives efficient estimates. As a future work, a Bayesian analysis via another approximation such as Laplace approximation method or Markov chain Monte Carlo (MCMC) algorithm will be discussed. 
Bayesian Estimation under Constant-Stress Partially Accelerated Life Test ...

$\mathrm{T}$ a b 1 e 1

Results of MLEs and Approximate BEs with Corresponding Estimated Variances and MSEs $(\beta=3, \theta=0.8, \alpha=0.5, \pi=0.50$, and $\eta=10)$ Using Different Time-Censored Sample Sizes

\begin{tabular}{|c|c|c|c|c|c|}
\hline$n$ & Parameter & Method & Estimate & MSE & Variance \\
\hline \multirow[t]{3}{*}{25} & $\beta$ & $\begin{array}{c}\text { ML } \\
\text { Bayes }\end{array}$ & $\begin{array}{l}3.6014 \\
3.4712 \\
\end{array}$ & $\begin{array}{l}0.0692 \\
0.0586\end{array}$ & $\begin{array}{l}0.0372 \\
0.0295\end{array}$ \\
\hline & $\theta$ & $\begin{array}{c}\text { ML } \\
\text { Bayes }\end{array}$ & $\begin{array}{l}1.2431 \\
0.9374 \\
\end{array}$ & $\begin{array}{l}0.0396 \\
0.0298 \\
\end{array}$ & $\begin{array}{l}0.0166 \\
0.0082\end{array}$ \\
\hline & $\alpha$ & $\begin{array}{c}\text { ML } \\
\text { Bayes }\end{array}$ & $\begin{array}{l}0.8211 \\
0.7855 \\
\end{array}$ & $\begin{array}{l}0.0286 \\
0.0214 \\
\end{array}$ & $\begin{array}{l}0.0074 \\
0.0041\end{array}$ \\
\hline \multirow[t]{3}{*}{50} & $\beta$ & $\begin{array}{c}\text { ML } \\
\text { Bayes }\end{array}$ & $\begin{array}{l}3.3862 \\
3.2281\end{array}$ & $\begin{array}{l}0.0509 \\
0.0389 \\
\end{array}$ & $\begin{array}{l}0.0242 \\
0.0148\end{array}$ \\
\hline & $\theta$ & $\begin{array}{c}\text { ML } \\
\text { Bayes }\end{array}$ & $\begin{array}{l}0.9747 \\
0.8911 \\
\end{array}$ & $\begin{array}{l}0.0274 \\
0.0185 \\
\end{array}$ & $\begin{array}{l}0.0096 \\
0.0051\end{array}$ \\
\hline & $\alpha$ & $\begin{array}{c}\text { ML } \\
\text { Bayes }\end{array}$ & $\begin{array}{l}0.6733 \\
0.6209 \\
\end{array}$ & $\begin{array}{l}0.0211 \\
0.0150\end{array}$ & $\begin{array}{l}0.0033 \\
0.0015\end{array}$ \\
\hline \multirow[t]{3}{*}{75} & $\beta$ & $\begin{array}{c}\text { ML } \\
\text { Bayes }\end{array}$ & $\begin{array}{l}3.2911 \\
3.0766 \\
\end{array}$ & $\begin{array}{l}0.0361 \\
0.0302 \\
\end{array}$ & $\begin{array}{l}0.0124 \\
0.0043\end{array}$ \\
\hline & $\theta$ & $\begin{array}{c}\text { ML } \\
\text { Bayes }\end{array}$ & $\begin{array}{l}0.8823 \\
0.8477 \\
\end{array}$ & $\begin{array}{l}0.0201 \\
0.0155 \\
\end{array}$ & $\begin{array}{l}0.0038 \\
0.0023 \\
\end{array}$ \\
\hline & $\alpha$ & $\begin{array}{c}\text { ML } \\
\text { Bayes }\end{array}$ & $\begin{array}{l}0.5725 \\
0.5410 \\
\end{array}$ & $\begin{array}{l}0.0048 \\
0.0019 \\
\end{array}$ & $\begin{array}{l}0.0011 \\
0.0006\end{array}$ \\
\hline \multirow[t]{3}{*}{100} & $\beta$ & $\begin{array}{c}\text { ML } \\
\text { Bayes }\end{array}$ & $\begin{array}{l}3.1208 \\
3.0241 \\
\end{array}$ & $\begin{array}{l}0.0069 \\
0.0038 \\
\end{array}$ & $\begin{array}{l}0.0025 \\
0.0023 \\
\end{array}$ \\
\hline & $\theta$ & $\begin{array}{c}\text { ML } \\
\text { Bayes }\end{array}$ & $\begin{array}{l}0.8351 \\
0.8126 \\
\end{array}$ & $\begin{array}{l}0.0054 \\
0.0030\end{array}$ & $\begin{array}{l}0.0022 \\
0.0008\end{array}$ \\
\hline & $\alpha$ & $\begin{array}{c}\text { ML } \\
\text { Bayes }\end{array}$ & $\begin{array}{l}0.5219 \\
0.5046\end{array}$ & $\begin{array}{l}0.0015 \\
0.0013\end{array}$ & $\begin{array}{l}0.0005 \\
0.0003\end{array}$ \\
\hline
\end{tabular}

$\mathrm{T}$ a b 1 e 2

Results of MLEs and Approximate BEs with Corresponding Estimated Variances and MSEs $(\beta=2, \theta=1.2, \alpha=1.5, \pi=0.50$, and $\eta=10)$ Using Different Time-Censored Sample Sizes

\begin{tabular}{|c|c|c|c|c|c|}
\hline$n$ & Parameter & Method & Estimate & MSE & Variance \\
\hline \multirow[t]{3}{*}{25} & $\beta$ & $\begin{array}{c}\text { ML } \\
\text { Bayes }\end{array}$ & $\begin{array}{l}2.5233 \\
2.4072\end{array}$ & $\begin{array}{l}0.0436 \\
0.0369\end{array}$ & $\begin{array}{l}0.0212 \\
0.0168\end{array}$ \\
\hline & $\theta$ & $\begin{array}{c}\text { ML } \\
\text { Bayes }\end{array}$ & $\begin{array}{l}1.4371 \\
1.3642\end{array}$ & $\begin{array}{l}0.0249 \\
0.0188\end{array}$ & $\begin{array}{l}0.0095 \\
0.0047\end{array}$ \\
\hline & $\alpha$ & $\begin{array}{c}\text { ML } \\
\text { Bayes }\end{array}$ & $\begin{array}{l}1.7648 \\
1.6427\end{array}$ & $\begin{array}{l}0.0180 \\
0.0135\end{array}$ & $\begin{array}{l}0.0042 \\
0.0023\end{array}$ \\
\hline \multirow[t]{3}{*}{50} & $\beta$ & $\begin{array}{c}\text { ML } \\
\text { Bayes }\end{array}$ & $\begin{array}{l}2.3977 \\
2.3104 \\
\end{array}$ & $\begin{array}{l}0.0321 \\
0.0245\end{array}$ & $\begin{array}{l}0.0138 \\
0.0084\end{array}$ \\
\hline & $\theta$ & $\begin{array}{c}\text { ML } \\
\text { Bayes }\end{array}$ & $\begin{array}{l}1.2894 \\
1.2380 \\
\end{array}$ & $\begin{array}{l}0.0173 \\
0.0117 \\
\end{array}$ & $\begin{array}{l}0.0055 \\
0.0029 \\
\end{array}$ \\
\hline & $\alpha$ & $\begin{array}{c}\text { ML } \\
\text { Bayes }\end{array}$ & $\begin{array}{l}1.5876 \\
1.5392 \\
\end{array}$ & $\begin{array}{l}0.0133 \\
0.0095 \\
\end{array}$ & $\begin{array}{l}0.0019 \\
0.0009 \\
\end{array}$ \\
\hline \multirow[t]{3}{*}{75} & $\beta$ & $\begin{array}{c}\text { ML } \\
\text { Bayes }\end{array}$ & $\begin{array}{l}2.1247 \\
2.0486 \\
\end{array}$ & $\begin{array}{l}0.0227 \\
0.0192 \\
\end{array}$ & $\begin{array}{l}0.0071 \\
0.0025 \\
\end{array}$ \\
\hline & $\theta$ & $\begin{array}{c}\text { ML } \\
\text { Bayes }\end{array}$ & $\begin{array}{l}1.2432 \\
1.2211 \\
\end{array}$ & $\begin{array}{l}0.0127 \\
0.0098 \\
\end{array}$ & $\begin{array}{l}0.0022 \\
0.0013\end{array}$ \\
\hline & $\alpha$ & $\begin{array}{c}\text { ML } \\
\text { Bayes }\end{array}$ & $\begin{array}{l}1.5333 \\
1.5104 \\
\end{array}$ & $\begin{array}{l}0.0032 \\
0.0012 \\
\end{array}$ & $\begin{array}{l}0.0006 \\
0.0002\end{array}$ \\
\hline \multirow[t]{3}{*}{100} & $\beta$ & $\begin{array}{c}\text { ML } \\
\text { Bayes }\end{array}$ & $\begin{array}{l}2.0394 \\
2.0113 \\
\end{array}$ & $\begin{array}{l}0.0043 \\
0.0024\end{array}$ & $\begin{array}{l}0.0014 \\
0.0011 \\
\end{array}$ \\
\hline & $\theta$ & $\begin{array}{c}\text { ML } \\
\text { Bayes }\end{array}$ & $\begin{array}{l}1.2117 \\
1.2021 \\
\end{array}$ & $\begin{array}{l}0.0034 \\
0.0019 \\
\end{array}$ & $\begin{array}{l}0.0013 \\
0.0005 \\
\end{array}$ \\
\hline & $\alpha$ & $\begin{array}{c}\text { ML } \\
\text { Bayes }\end{array}$ & $\begin{array}{l}1.5102 \\
1.5002 \\
\end{array}$ & $\begin{array}{l}0.0009 \\
0.0008 \\
\end{array}$ & $\begin{array}{l}0.0003 \\
0.0001 \\
\end{array}$ \\
\hline
\end{tabular}


T a b 1 e 3

Results of MLEs and Approximate BEs with Corresponding Estimated Variances and MSEs $(\beta=3, \theta=0.8, \alpha=0.5, \pi=0.30$, and $\eta=10)$ Using Different Time-Censored Sample Sizes

\begin{tabular}{|c|c|c|c|c|c|}
\hline$n$ & Parameter & Method & Estimate & MSE & Variance \\
\hline \multirow[t]{3}{*}{25} & $\beta$ & $\begin{array}{c}\text { ML } \\
\text { Bayes }\end{array}$ & $\begin{array}{l}.9125 \\
3.5824 \\
\end{array}$ & $\begin{array}{l}0.0985 \\
0.0779\end{array}$ & $\begin{array}{l}0.0502 \\
0.0398 \\
\end{array}$ \\
\hline & $\theta$ & $\begin{array}{c}\text { ML } \\
\text { Bayes }\end{array}$ & $\begin{array}{l}1.4729 \\
1.2366 \\
\end{array}$ & $\begin{array}{l}0.0675 \\
0.0533 \\
\end{array}$ & $\begin{array}{l}0.0224 \\
0.0111 \\
\end{array}$ \\
\hline & $\alpha$ & $\begin{array}{c}\text { ML } \\
\text { Bayes }\end{array}$ & $\begin{array}{l}1.0781 \\
1.0262 \\
\end{array}$ & $\begin{array}{l}0.0492 \\
0.0346 \\
\end{array}$ & $\begin{array}{l}0.0102 \\
0.0055\end{array}$ \\
\hline \multirow[t]{3}{*}{50} & $\beta$ & $\begin{array}{c}\text { ML } \\
\text { Bayes }\end{array}$ & $\begin{array}{l}3.4521 \\
3.3217 \\
\end{array}$ & $\begin{array}{l}0.0665 \\
0.0492 \\
\end{array}$ & $\begin{array}{l}0.0327 \\
0.0203 \\
\end{array}$ \\
\hline & $\theta$ & $\begin{array}{c}\text { ML } \\
\text { Bayes }\end{array}$ & $\begin{array}{l}1.2107 \\
1.1638 \\
\end{array}$ & $\begin{array}{l}0.0477 \\
0.0314 \\
\end{array}$ & $\begin{array}{l}0.0131 \\
0.0069 \\
\end{array}$ \\
\hline & $\alpha$ & $\begin{array}{c}\text { ML } \\
\text { Bayes }\end{array}$ & $\begin{array}{l}0.9658 \\
0.9104 \\
\end{array}$ & $\begin{array}{l}0.0311 \\
0.0294 \\
\end{array}$ & $\begin{array}{l}0.0045 \\
0.0026 \\
\end{array}$ \\
\hline \multirow[t]{3}{*}{75} & $\beta$ & $\begin{array}{c}\text { ML } \\
\text { Bayes }\end{array}$ & $\begin{array}{l}3.2982 \\
3.2290 \\
\end{array}$ & $\begin{array}{l}0.0431 \\
0.0378 \\
\end{array}$ & $\begin{array}{l}0.0167 \\
0.0058 \\
\end{array}$ \\
\hline & $\theta$ & $\begin{array}{c}\text { ML } \\
\text { Bayes }\end{array}$ & $\begin{array}{l}1.0726 \\
1.0179 \\
\end{array}$ & $\begin{array}{l}0.0287 \\
0.0212 \\
\end{array}$ & $\begin{array}{l}0.0051 \\
0.0031 \\
\end{array}$ \\
\hline & $\alpha$ & $\begin{array}{c}\text { ML } \\
\text { Bayes }\end{array}$ & $\begin{array}{l}0.8721 \\
0.7913 \\
\end{array}$ & $\begin{array}{l}0.0113 \\
0.0102 \\
\end{array}$ & $\begin{array}{l}0.0015 \\
0.0008 \\
\end{array}$ \\
\hline \multirow[t]{3}{*}{100} & $\beta$ & $\begin{array}{c}\text { ML } \\
\text { Bayes }\end{array}$ & $\begin{array}{l}3.1876 \\
3.1155 \\
\end{array}$ & $\begin{array}{l}0.0094 \\
0.0057 \\
\end{array}$ & $\begin{array}{l}0.0034 \\
0.0031 \\
\end{array}$ \\
\hline & $\theta$ & $\begin{array}{c}\text { ML } \\
\text { Bayes }\end{array}$ & $\begin{array}{l}0.9857 \\
0.9274 \\
\end{array}$ & $\begin{array}{l}0.0088 \\
0.0067 \\
\end{array}$ & $\begin{array}{l}0.0030 \\
0.0011 \\
\end{array}$ \\
\hline & $\alpha$ & $\begin{array}{c}\text { ML } \\
\text { Bayes }\end{array}$ & $\begin{array}{l}0.7119 \\
0.6781 \\
\end{array}$ & $\begin{array}{l}0.0052 \\
0.0034 \\
\end{array}$ & $\begin{array}{l}0.0007 \\
0.0004\end{array}$ \\
\hline
\end{tabular}

$\mathrm{T}$ a b 1 e 4

Results of MLEs and Approximate BEs with Corresponding Estimated Variances and MSEs $(\beta=2, \theta=1.2, \alpha=1.5, \pi=0.70$, and $\eta=10)$ Using Different Time-Censored Sample Sizes

\begin{tabular}{|c|c|c|c|c|c|}
\hline$n$ & Parameter & Method & Estimate & MSE & Variance \\
\hline \multirow[t]{3}{*}{25} & $\beta$ & $\begin{array}{c}\text { ML } \\
\text { Bayes }\end{array}$ & $\begin{array}{l}2.4113 \\
2.3271 \\
\end{array}$ & $\begin{array}{l}0.0379 \\
0.0321\end{array}$ & $\begin{array}{l}0.0187 \\
0.0148 \\
\end{array}$ \\
\hline & $\theta$ & $\begin{array}{c}\text { ML } \\
\text { Bayes }\end{array}$ & $\begin{array}{l}1.3570 \\
1.3111 \\
\end{array}$ & $\begin{array}{l}0.0217 \\
0.0164\end{array}$ & $\begin{array}{l}0.0084 \\
0.0041\end{array}$ \\
\hline & $\alpha$ & $\begin{array}{c}\text { ML } \\
\text { Bayes }\end{array}$ & $\begin{array}{l}1.7142 \\
1.6281 \\
\end{array}$ & $\begin{array}{l}0.0157 \\
0.0117 \\
\end{array}$ & $\begin{array}{l}0.0037 \\
0.0021 \\
\end{array}$ \\
\hline \multirow[t]{3}{*}{50} & $\beta$ & $\begin{array}{c}\text { ML } \\
\text { Bayes }\end{array}$ & $\begin{array}{l}2.2915 \\
2.2681 \\
\end{array}$ & $\begin{array}{l}0.0279 \\
0.0213 \\
\end{array}$ & $\begin{array}{l}0.0121 \\
0.0074 \\
\end{array}$ \\
\hline & $\theta$ & $\begin{array}{c}\text { ML } \\
\text { Bayes }\end{array}$ & $\begin{array}{l}1.2270 \\
1.1860 \\
\end{array}$ & $\begin{array}{l}0.0151 \\
0.0102 \\
\end{array}$ & $\begin{array}{l}0.0048 \\
0.0026\end{array}$ \\
\hline & $\alpha$ & $\begin{array}{c}\text { ML } \\
\text { Bayes }\end{array}$ & $\begin{array}{l}1.5852 \\
1.5472 \\
\end{array}$ & $\begin{array}{l}0.0116 \\
0.0083 \\
\end{array}$ & $\begin{array}{l}0.0017 \\
0.0008 \\
\end{array}$ \\
\hline \multirow[t]{3}{*}{75} & $\beta$ & $\begin{array}{c}\text { ML } \\
\text { Bayes }\end{array}$ & $\begin{array}{l}2.0844 \\
2.0352 \\
\end{array}$ & $\begin{array}{l}0.0197 \\
0.0167\end{array}$ & $\begin{array}{l}0.0062 \\
0.0022 \\
\end{array}$ \\
\hline & $\theta$ & $\begin{array}{c}\text { ML } \\
\text { Bayes }\end{array}$ & $\begin{array}{l}1.2130 \\
1.1941 \\
\end{array}$ & $\begin{array}{l}0.0112 \\
0.0085\end{array}$ & $\begin{array}{l}0.0019 \\
0.0012 \\
\end{array}$ \\
\hline & $\alpha$ & $\begin{array}{c}\text { ML } \\
\text { Bayes }\end{array}$ & $\begin{array}{l}1.5318 \\
1.5009 \\
\end{array}$ & $\begin{array}{l}0.0028 \\
0.0014\end{array}$ & $\begin{array}{l}0.0005 \\
0.0002 \\
\end{array}$ \\
\hline \multirow[t]{3}{*}{100} & $\beta$ & $\begin{array}{c}\text { ML } \\
\text { Bayes }\end{array}$ & $\begin{array}{l}2.0102 \\
2.0087\end{array}$ & $\begin{array}{l}0.0037 \\
0.0021\end{array}$ & $\begin{array}{l}0.0012 \\
0.0010\end{array}$ \\
\hline & $\theta$ & $\begin{array}{c}\text { ML } \\
\text { Bayes }\end{array}$ & $\begin{array}{l}1.2024 \\
1.2007 \\
\end{array}$ & $\begin{array}{l}0.0032 \\
0.0017 \\
\end{array}$ & $\begin{array}{l}0.0011 \\
0.0004 \\
\end{array}$ \\
\hline & $\alpha$ & $\begin{array}{c}\text { ML } \\
\text { Bayes }\end{array}$ & $\begin{array}{l}1.5001 \\
1.5000 \\
\end{array}$ & $\begin{array}{l}0.0007 \\
0.0005 \\
\end{array}$ & $\begin{array}{l}0.0002 \\
0.0001 \\
\end{array}$ \\
\hline
\end{tabular}


APPENDIX (Derivation of Posterior Means and Posterior Variances):

Here, there are three parameters in the model. That is, $m=3$. Let the subscripts 1,2 , and 3 refer to $\beta, \theta$, and $\alpha$, respectively. It is not easy to obtain the posterior moments analytically. Therefore, using Lindley expansion, the posterior mean (i.e., Bayesian estimator under squared-error loss function) and the posterior variance of $\beta$ are given, respectively, in the form

$$
\beta^{*}=E(\beta \mid y)=\left[\beta-\left(\frac{\sigma_{11}}{\beta}+\frac{\sigma_{12}}{\theta}+\frac{\sigma_{13}}{\alpha}\right)+\frac{1}{2}\left(\sigma_{11} E_{1}+\sigma_{12} E_{2}+\sigma_{13} E_{3}\right)\right] \downarrow \hat{\Theta},
$$

and

$$
\begin{gathered}
\operatorname{var}(\beta \mid y)=E\left(\beta^{2} \mid y\right)-\left(\beta^{*}\right)^{2}= \\
=\sigma_{11}-\left[\left(\frac{\sigma_{11}}{\beta}+\frac{\sigma_{12}}{\theta}+\frac{\sigma_{13}}{\alpha}\right)-\frac{1}{2}\left(\sigma_{11} E_{1}+\sigma_{12} E_{2}+\sigma_{13} E_{3}\right)\right]^{2} \downarrow \hat{\Theta} .
\end{gathered}
$$

Applying the same technique, the posterior mean and posterior variance of the scale parameter $\theta$ take the following form:

$$
\theta^{*}=E(\theta \mid y)=\left[\theta-\left(\frac{\sigma_{21}}{\beta}+\frac{\sigma_{22}}{\theta}+\frac{\sigma_{23}}{\alpha}\right)+\frac{1}{2}\left(\sigma_{21} E_{1}+\sigma_{22} E_{2}+\sigma_{23} E_{3}\right)\right] \downarrow \hat{\Theta},
$$

and

$$
\operatorname{var}(\theta \mid y)=\sigma_{22}-\left[\left(\frac{\sigma_{21}}{\beta}+\frac{\sigma_{22}}{\theta}+\frac{\sigma_{23}}{\alpha}\right)-\frac{1}{2}\left(\sigma_{21} E_{1}+\sigma_{22} E_{2}+\sigma_{23} E_{3}\right)\right]^{2} \downarrow \hat{\Theta} .
$$

Similarly, for the shape parameter $\alpha$, the posterior mean and the posterior variance are given by

$$
\alpha^{*}=E(\alpha \mid y)=\left[\alpha-\left(\frac{\sigma_{31}}{\beta}+\frac{\sigma_{32}}{\theta}+\frac{\sigma_{33}}{\alpha}\right)+\frac{1}{2}\left(\sigma_{31} E_{1}+\sigma_{32} E_{2}+\sigma_{33} E_{3}\right)\right] \downarrow \hat{\Theta},
$$

and

$$
\operatorname{var}(\alpha \mid y)=\sigma_{33}-\left[\left(\frac{\sigma_{31}}{\beta}+\frac{\sigma_{32}}{\theta}+\frac{\sigma_{33}}{\alpha}\right)-\frac{1}{2}\left(\sigma_{31} E_{1}+\sigma_{32} E_{2}+\sigma_{33} E_{3}\right)\right]^{2} \downarrow \hat{\Theta},
$$

where

$$
E_{1}=\sum_{i, j} \sigma_{i j} L_{i j 1}^{(3)}, \quad E_{2}=\sum_{i, j} \sigma_{i j} L_{i j 2}^{(3)}, \quad E_{3}=\sum_{i, j} \sigma_{i j} L_{i j 3}^{(3)},
$$

for $i, j=1,2,3, \sigma_{i j}$ are the elements of the inverse of the asymptotic Fisher-information matrix of the ML estimators of $\beta, \theta$, and $\alpha$ in the case of type-I censored data and $i, j=1,2,3$, is the third derivatives of the $L_{i j k}^{(3)}$ natural logarithm of the likelihood function in type-I censoring.

To compute the posterior means and the posterior variances of $\beta, \theta$, and $\alpha$ derived before, both second and third derivatives of the natural logarithm of the likelihood function in (7) must be got. 
The second derivatives can be given by the following equations:

$$
\begin{gathered}
\frac{\partial^{2} \ln L}{\partial \beta^{2}}=-\frac{n_{a}}{\beta^{2}}+\frac{\left(n \pi-n_{a}\right) \alpha \eta^{2}}{(\theta+\beta \eta)^{2}}+(\alpha+1) \sum_{j=1}^{n \pi} \delta_{a j} \frac{x_{j}^{2}}{\left(\theta+\beta x_{j}\right)^{2}}, \\
\frac{\partial^{2} \ln L}{\partial \beta \partial \theta}=\frac{\left(n \pi-n_{a}\right) \alpha \eta}{(\theta+\beta \eta)^{2}}+(\alpha+1) \sum_{j=1}^{n \pi} \delta_{a j} \frac{x_{j}}{\left(\theta+\beta x_{j}\right)^{2}}, \\
\frac{\partial^{2} \ln L}{\partial \beta \partial \alpha}=-\frac{\left(n \pi-n_{a}\right) \eta}{\theta+\beta \eta}-\sum_{j=1}^{n \pi} \delta_{a j} \frac{x_{j}}{\theta+\beta x_{j}}, \\
\frac{\partial^{2} \ln L}{\theta^{2}}=-\frac{n \alpha}{\theta^{2}}+\frac{\left(n \pi-n_{a}\right) \alpha}{(\theta+\beta \eta)^{2}}+\frac{\left(n \bar{\pi}-n_{u}\right) \alpha}{(\theta+\eta)^{2}}+(\alpha+1)\left[\sum_{i=1}^{n \bar{\pi}} \frac{\partial_{u i}}{\left(\theta+t_{i}\right)^{2}}+\sum_{j=1}^{n \pi} \frac{\delta_{a j}}{\left(\theta+\beta x_{j}\right)^{2}}\right], \\
\frac{\partial^{2} \ln L}{\partial \theta \partial \alpha}=\frac{n}{\theta}-\frac{\left(n \pi-n_{a}\right)}{\theta+\beta \eta}-\frac{\left(n \bar{\pi}-n_{u}\right)}{\theta+\eta}-\left[\sum_{i=1}^{n \pi \bar{\pi}} \frac{\delta_{u i}}{\theta+t_{i}}+\sum_{j=1}^{n \pi} \frac{\delta_{a j}}{\theta+\beta x_{j}}\right] \\
\frac{\partial^{2} \ln L}{\partial \alpha^{2}}=-\frac{n_{u}+n_{a}}{\alpha^{2}}
\end{gathered}
$$

For the third derivatives, they are given as follows:

$$
\begin{gathered}
L_{111}^{(3)}=\frac{\partial^{3} \ln L}{\partial \beta^{3}}=\frac{2 n_{a}}{\beta^{3}}-\frac{2\left(n \pi-n_{a}\right) \alpha \eta^{3}}{(\theta+\beta \eta)^{3}}-2(\alpha+1) \sum_{j=1}^{n \pi} \delta_{a j} \frac{x_{j}^{3}}{\left(\theta+\beta x_{j}\right)^{3}}, \\
L_{222}^{(3)}=\frac{\partial^{3} \ln L}{\partial \theta^{3}}=\frac{2 n \alpha}{\theta^{3}}-\frac{2\left(n \pi-n_{a}\right) \alpha}{(\theta+\beta \eta)^{3}}-\frac{2\left(n \bar{\pi}-n_{u}\right) \alpha}{(\theta+\eta)^{3}}- \\
-2(\alpha+1)\left[\sum_{i=1}^{n \bar{\pi}} \frac{\delta_{u i}}{\left(\theta+t_{i}\right)^{3}}+\sum_{j=1}^{n \pi} \frac{\delta_{a j}}{\left(\theta+\beta x_{j}\right)^{3}}\right] \\
L_{333}^{(3)}=\frac{\partial^{3} \ln L}{\partial \alpha^{3}}=\frac{2\left(n_{u}+n_{a}\right)}{\alpha^{3}}, \\
L_{112}^{(3)}=\frac{\partial^{3} \ln L}{\partial \beta^{2} \partial \theta}=-\frac{2\left(n \pi-n_{a}\right) \alpha \eta^{2}}{(\theta+\beta \eta)^{3}}-2(\alpha+1) \sum_{j=1}^{n \pi} \delta_{a j} \frac{x_{j}^{2}}{\left(\theta+\beta x_{j}\right)^{3}}=L_{121}^{(3)}=L_{211}^{(3)}, \\
L_{221}^{(3)}=\frac{\partial^{3} \ln L}{\partial \theta^{2} \partial \beta}=-\frac{2\left(n \pi-n_{a}\right) \alpha \eta}{(\theta+\beta \eta)^{3}}-2(\alpha+1) \sum_{j=1}^{n \pi} \delta_{a j} \frac{x_{j}}{\left(\theta+\beta x_{j}\right)^{3}}=L_{212}^{(3)}=L_{122}^{(3)},
\end{gathered}
$$




$$
\begin{gathered}
L_{113}^{(3)}=\frac{\partial^{3} \ln L}{\partial \beta^{2} \partial \alpha}=\frac{\left(n \pi-n_{a}\right) \eta^{2}}{(\theta+\beta \eta)^{2}}+\sum_{j=1}^{n \pi} \delta_{a j} \frac{x_{j}^{2}}{\left(\theta+\beta x_{j}\right)^{2}}=L_{131}^{(3)}=L_{311}^{(3)}, \\
L_{123}^{(3)}=\frac{\partial^{3} \ln L}{\partial \beta \partial \theta \partial \alpha}=\frac{\left(n \pi-n_{a}\right) \eta}{(\theta+\beta \eta)^{2}}+\sum_{j=1}^{n \pi} \delta_{a j} \frac{x_{j}}{\left(\theta+\beta x_{j}\right)^{2}}= \\
=L_{132}^{(3)}=L_{213}^{(3)}=L_{231}^{(3)}=L_{312}^{(3)}=L_{321}^{(3)}, \\
L_{223}^{(3)}=\frac{\partial^{3} \ln L}{\partial \theta^{2} \partial \alpha}=-\frac{n}{\theta^{2}}+\frac{\left(n \pi-n_{a}\right)}{(\theta+\beta \eta)^{2}}+\frac{\left(n \bar{\pi}-n_{u}\right)}{(\theta+\eta)^{2}}+ \\
+\sum_{i=1}^{n \bar{\pi}} \frac{\delta_{u i}}{\left(\theta+t_{i}\right)^{2}}+\sum_{j=1}^{n \pi} \frac{\delta_{a j}}{\left(\theta+\beta x_{j}\right)^{2}}=L_{232}^{(3)}=L_{322}^{(3)}, \\
L_{331}^{(3)}=\frac{\partial^{3} \ln L}{\partial \alpha^{2} \partial \beta}=L_{313}^{(3)}=L_{133}^{(3)}, \\
L_{332}^{(3)}=\frac{\partial^{3} \ln L}{\partial \alpha^{2} \partial \theta}=L_{323}^{(3)}=L_{233}^{(3)} .
\end{gathered}
$$

Acknowledgments. This project was supported by King Saud University, Deanship of Scientific Research, College of Science Research Center.

\section{Резиме}

Досліджуються ймовірність параметрів моделі і використання методу Байеса для моделі частково прискорених ресурсних випробувань при постійних навантаженнях і цензуруванні в часі типу I для розподілу Парето другого роду. Виконано розрахунок максимальної ймовірності й індикаторів Байеса для параметрів моделі. За допомогою методу апроксимації Ліндлі отримано апостеріорні середні значення і варіації для квадратичної функції втрат (помилок). Показано переваги даної апроксимації. Виконано чисельне моделювання за методом Монте-Карло для зразків різного розміру $\mathrm{i}$ параметрів моделі для порівняльної оцінки запропонованих методів прогнозування pecypcy.

1. W. Nelson, Accelerated Testing: Statistical Models, Test Plans, and Data Analysis, Wiley, New York (1990).

2. Ali A. Ismail, "Estimating the generalized exponential distribution parameters and the acceleration factor under constant-stress partially accelerated life testing with type-II censoring," Strength Mater., 45, No. 6, 693-702 (2013).

3. D. S. Bai and S. W. Chung, "Optimal design of partially accelerated life tests for the exponential distribution under type-I censoring," IEEE Trans. Reliab., 41, No. 3, 400-406 (1992).

4. D. S. Bai, D. S. W. Chung, and Y. R. Chun, "Optimal design of partially accelerated life tests for the lognormal distribution under type-I censoring," Reliab. Eng. Syst. Safety, 40, No. 1, 85-92 (1993). 
5. A. A. Ismail, A. A. Abdel-Ghaly, and E. H. El-Khodary, "Optimum constant-stress life test plans for Pareto distribution under type-I censoring," J. Stat. Comput. Simul., 81, No. 12, 1835-1845 (2011).

6. Ali A. Ismail, "On designing constant-stress partially accelerated life tests under time-censoring," Strength Mater., 46, No. 1, 132-139 (2014).

7. S. K. Sinha, Reliability and Life Testing, Wiley, New York (1986).

8. J. A. Achcar, "Approximate Bayesian inference for accelerated life tests," J. Appl. Stat. Sci., 1, No. 3, 223-237 (1994).

9. P. K. Goel, Some Estimation Problems in the Study of Tampered Random Variables, Technical Report No. 50, Department of Statistics, Carnegie-Mellon University, Pittsburgh, PA (1971).

10. M. H. DeGroot and P. K. Goel, "Bayesian and optimal design in partially accelerated life testing," Naval Res. Logist. Quart., 16, No. 2, 223-235 (1979).

11. M. M. Abdel-Ghani, Investigation of Some Lifetime Models under Partially Accelerated Life Tests, Ph.D. Thesis, Department of Statistics, Faculty of Economics and Political Science, Cairo University, Giza, Egypt (1998).

12. A. A. Ismail, "Bayes estimation of gompertz distribution parameters and acceleration factor under partially accelerated life tests with type-I censoring," J. Stat. Comput. Simul., 80, No. 11, 1253-1264 (2010).

13. D. V. Lindley, "Approximate Bayesian methods," Trabajos Estadistica, 31, 223-237 (1980).

14. J. Green, "Discussant on D. V. Lindley's (1980) paper on approximate Bayesian methods," Trabajos Estadistica, 31, 241-243 (1980). 